\title{
Prediction of Joint Strength and Effect of the Surface Treatment on the Single Overlap Adhesive Joints
}

\author{
Filipe A. G. Giesteira ${ }^{1}$, E. A. S. Marques², R. J. C. Carbas³ , L. F. M. da \\ Silva ${ }^{4}$ \\ ${ }^{1}$ Department of Mechanical Engineering, Faculty of Engineering University of Porto, Porto, \\ Portugal (up201306293@fe.up.pt) ORCID 0000-0002-4901-2569; ${ }^{2}$ INEGI - Institute of Science \\ and Innovation in Mechanical and Industrial Engineering, Porto, Portugal \\ (emarques@fe.up.pt) ORCID 0000-0002-2750-8184; ${ }^{3}$ INEGI - Institute of Science and \\ Innovation in Mechanical and Industrial Engineering, Porto, Portugal (carbas@fe.up.pt) ORCID \\ 0000-0002-1933-0865; ${ }^{4}$ Department of Mechanical Engineering, Faculty of Engineering \\ University of Porto, Porto, Portugal (lucas@fe.up.pt) ORCID 0000-0003-3272-4591
}

\begin{abstract}
A two-component high-ductility adhesive (acrylic and catalyst based), SikaFast ${ }^{\circledR}$ 5211 NT, was used to bond single overlap joints with mild steel adherends and 25 $\mathrm{mm}$ of overlap. One joint configuration used treated bonding surfaces while the other was did not employ treatment of the adherend surfaces, with the aim of studying the influence of the material surface treatment. The specimens were tensile tested in a INSTRON ${ }^{\circledR}$ universal testing machine and the non-treated surface have shown a strength four times lower than the treated surface. Several analytical methods were used to predict joint strength, with two methods achieving reasonably accurate failure load predictions.
\end{abstract}

Author Keywords. Structural Adhesive Joints, Overlap Joints, Single Overlap Joints, Strength Prediction, Surface Preparation

Type: Research Article

○ Open Access $\square$ Peer Reviewed @(i) cC BY

\section{Introduction}

More than ever, adhesive bonding is becoming an important solution for achieving permanent mechanical joints. Adhesive bonding enables the manufacture of products with clean and visually appealing joints (without outstanding features like bolts, welding marks, rivets, etc.), but perhaps their most important advantage is their ability to efficiently join two different materials. This is a critical factor in the manufacture of composite structures construction and it the increased use of these advanced materials has significantly expanded the use of structural adhesives. In addition, adhesives are also able to compensate for differences in thermal expansion, which is very advantageous for use in structures exposed to heat. Nevertheless, adhesive bonding also has some inconveniences and disadvantages. One of the most critical aspects of adhesive joints is their strong dependency on the surface preparation condition (da Silva, Magalhães, and Moura 2007). Due to its high surface tension, metals tend to absorb oils and contamination present in vapor form. If the aim is to increase long term durability several options are available such as using vapor degreasing with an organic solvent such as trichloroethane, sandblasting (increasing the adhesive contact surface by roughening the metal surface) and chemical etching, by removing all the weakly bonded oxides and forming strongly bonded ones. In order to design a strong and durable joint, it is necessary to take into account several aspects such as: adhesion theory, adhesive properties, joint 
modelling and surface preparation. The study presented in this document is related to the last two of these topics, focusing on the quality of surface preparation and its impact on the prediction of joint strength. The prediction of joint strength can be attained using many different models that try to quantitatively describe the mechanical behaviour of an adhesive bond. The models applied in this work are the shear lag analysis or analytical method of Volkersen (Volkersen 1938) (capable of modelling the elasticity of the adhesive material), the Goland and Reissner first approximation (Goland and Reissner 1944) (which does not neglect the joint rotation), the Generalized Failure Criterion (Hart-Smith 1973) (which takes in account of the ability of the adhesive material to withstand plastic deformation) and the adherend failure criterion (Adams, Comyn, and Wake 1997) which is based on the Goland and Reissner Theory and suitable for adherends that deform plastically. The following sections will detail the calculation associated with these methods.

\subsection{Volkersen's criterion for shear lag analysis}

The Volkersen model is an analytical method that assumes perfectly linear elastic behaviour from the adhesive bond and an interface continuity condition. The shear lag model or differential straining considers that the only mechanism of load transference from one adherend to another is a simple shearing mechanism (Gomes 2004; Adams, Comyn, and Wake 1997). Therefore, indirectly also imposes that:

- the adhesive deforms only in shear or,

- the adherend deforms only in tension.

These assumptions would be entirely true if the adhesive material had null ductility, or if the evaluation was purely made in the elastic domain. However, it is expected to be accurate in the presence of brittle adhesive materials, which is not the case.

The following equations model the shear stress distribution through the joint according to Volkersen.

$$
\frac{\tau}{\bar{\tau}}=\frac{\lambda l}{\varphi \sinh (\lambda l)}[(\phi-1) \cosh (\lambda(l-x))+\cosh (\lambda x)]
$$

With $\bar{\tau}, \phi$ and $\lambda$ each given by

$$
\begin{gathered}
\bar{\tau}=\frac{P}{b l} \\
\phi=\frac{E_{1} t_{1}}{E_{2} t_{2}}+1 \\
\lambda=\sqrt{\frac{G_{a}}{t_{a}}\left(\frac{1}{E_{1} t_{1}}+\frac{1}{E_{2} t_{2}}\right)}
\end{gathered}
$$

Note that the indexes 1 and 2 refer to the adherend relative position. The previous expressions where deduced admitting the hypothesis of different materials and geometry for each of the adherends. This model is schematically shown in Figure 1. 


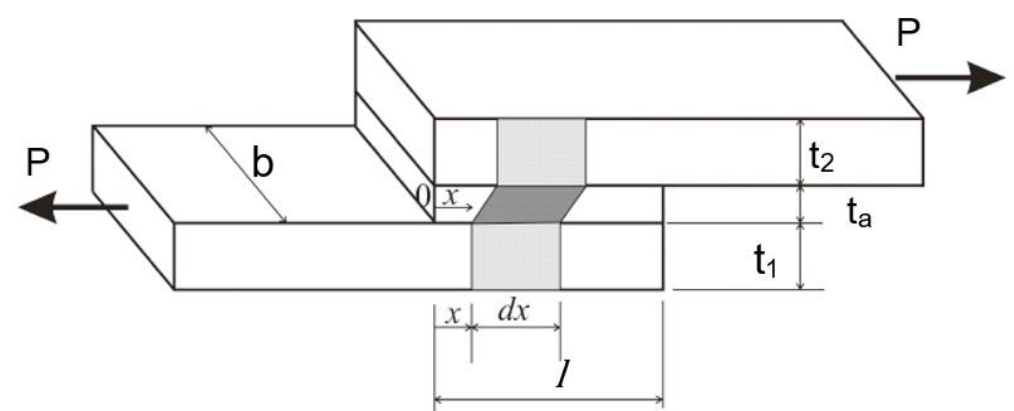

Figure 1: Model used by Volkersen to express the elastic behaviour of an overlap joint (da Silva, Öchsner, and Adams 2011)

However, for this particular situation $E_{1}=E_{2}$ and $t_{1}=t_{2}$ so (3) and (4) result in:

$$
\begin{gathered}
\phi=\frac{E_{1} t_{1}}{E_{2} t_{2}}+1=2 \\
\lambda=\sqrt{\frac{G_{a}}{t_{a}}\left(\frac{1}{E_{1} t_{1}}+\frac{1}{E_{2} t_{2}}\right)}=\sqrt{\frac{2 G_{a}}{t_{a} E_{M} t_{s}}}
\end{gathered}
$$

Since it is necessary to quantify the maximum load supported by the joint, it is advantageous to combine (1) with (2) and considering (5) all comes:

$$
P=\frac{\tau \cdot b \cdot 2 \sinh (\lambda l)}{\lambda \cdot[\cosh (\lambda(l-x))+\cosh (\lambda x)]}
$$

For the limit case $\tau=\tau_{y_{a}}$

$$
P=\frac{\tau_{y_{a}} \cdot b \cdot 2 \sinh (\lambda l)}{\lambda \cdot[\cosh (\lambda(l-x))+\cosh (\lambda x)]}
$$

Before determining the output of $\lambda$ it is necessary to estimate the shear modulus of the adhesive material $(G)$. For perfect isotropic materials, the parameters $G_{a}, K$, and $v$, are related by the following expression (Gomes 2004):

$$
2 G(1+v)=E=3 K(1-2 v)
$$

The materials that probably more closely approach the isotropic behaviour are polymers. Assuming an isotropic behaviour from the polymeric adhesive we can estimate $G$ from (9):

$$
G_{a}=\frac{E_{a}}{2 \cdot(1+v)} \approx 0.093 \mathrm{GPa}
$$

The results will be the same for each specimen, since no analytical criterion is capable of modelling an adhesion defect.

Knowing $G_{a}, \lambda$ can be calculated with

$$
\lambda=\sqrt{\frac{2 G_{a}}{t_{a} E_{M} t_{s}}}=47.06\left[\mathrm{~m}^{-1}\right]
$$

$P=f(x)$ will be plotted and calculated for the extremes

$$
P \max =\frac{\tau_{y} \cdot b \cdot 2 \sinh (\lambda l)}{\lambda \cdot[\cosh (\lambda(l-x))+\cosh (\lambda x)]}
$$




\subsection{Goland and Reissner theory}

The Goland and Reissner theory was devised to model the effect of the bending phenomenon that occurs during tensile testing of a single lap joint.

As shown in Figure 2, the stress state is more complex than that described by Volkersen. A bending moment and a transverse load must be taken in account.

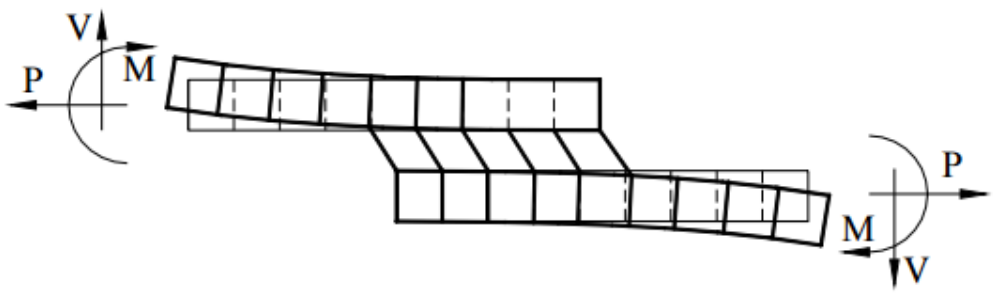

Figure 2: Schematic illustration of the bending moment effect over the single overlap joint. Adapted by the authors from Adams, Comyn, and Wake 1997

The existence of non-collinear loads is demonstrated in Figure 3.

a)

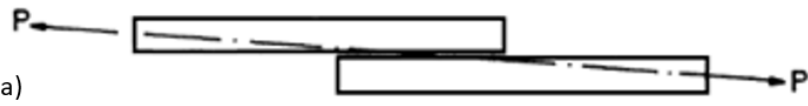

b)

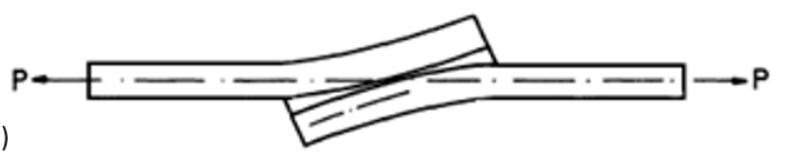

Figure 3: a): Undeformed single overlap joint, with exaggerated bending moment, resulting from the non-collinear loads. b) Geometrically representation of the Goland and Reissner bending moment factor. Adapted by the authors from Adams, Comyn, and Wake 1997

The bending momentum can be represented by the following equations.

$$
\begin{gathered}
M_{0}=\frac{P \cdot \delta}{2} \\
M=k \cdot \frac{P \cdot \delta}{2}
\end{gathered}
$$

In fact, the model described here consists on the first theory or approximation of Goland and Reissner. In order to be able of solving the nonlinear problem that the introduction of joint rotation yielded, two limiting cases were considered.

In the first case, the adhesive layer was considered to be of negligible thickness so that its effect on the joint flexibility can be neglected compared with that of the adherend thickness. In the second case, the joint flexibility was mainly due to the adhesive layer.

The following equations model the shear stress distribution on the adhesive material.

$$
\frac{\boldsymbol{\tau}(x)}{\overline{\boldsymbol{\tau}}}=\frac{1}{4}\left[\frac{\beta l}{2 t}(1+3 k) \cdot \frac{\cosh \left(\frac{\beta \mathrm{x}}{2 t}\right)}{\sinh \left(\frac{\beta l}{2 t}\right)}+3(1-k)\right]
$$

With:

$$
\beta=\frac{8 t G_{a}}{E t t_{a}}
$$




\subsection{Generalized failure criterion}

The Generalized Failure Criterion is a specific case of the Hart-Smith theory. For very ductile adhesives, it is a good approximation of the reality to assume that the whole adhesive length will deform plastically before adhesive failure occurs.

According to Hart-Smith, this assumption produces fairly good results for adhesives with more than $20 \%$ of ductility, which should be a good match for the ductile adhesive used in this work. The maximum load will be simply given by:

$$
P_{\max }=\tau_{y} b l
$$

\subsection{Adherend failure criterion}

In an attempt to cover all the aspects mentioned earlier, Adams, Comyn, and Wake (1997) devised a simple but robust strength prediction procedure. Their approach divides the problem in two extreme cases:

- For ductile adherends and adhesives the failure analysis is done in conjunction with the Goland and Reissner theory, assuming that the adherend is the weakest link in the chain.

- For stiff adherends and very ductile adhesives materials, the generalized failure criterion is used.

The overlap length parameter greatly influences this division. The higher the overlap length, the lower is the influence of the adhesive ductility. Figure 4 illustrates the relationship between overlap length, failure load and the locus of failure.

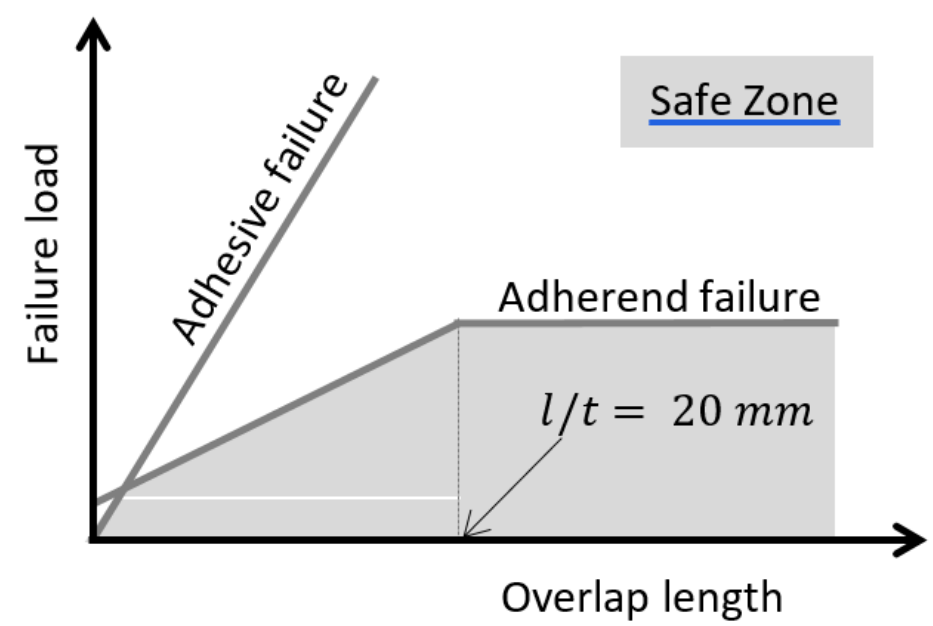

Figure 4: Schematic representation of the failure load as a function of the overlap length. Based on da Silva, Öchsner, and Adams (2011), adapted by the authors

This model resorted to the already known Goland and Reissner theory. Combining Equation 15 and the tension stress due to the P load with the Euler theory for simple bending elements,

$$
\sigma_{f}=\frac{6 M_{f}}{b t^{2}}
$$

Normal tension for a rectangular beam loaded with a bending moment. Comes:

$$
P_{\max }=\frac{\sigma_{y} \cdot b t}{1+3 k b}
$$


Where $\mathrm{k}$ is given by the Goland and Reissner theory by:

$$
k=\frac{\cosh \left(u_{2} \frac{l}{2}\right)}{\cosh \left(u_{2} \frac{l}{2}\right)+2 \sqrt{2} \cdot \sinh \left(u_{2} \frac{l}{2}\right)}
$$

with,

$$
u_{2}=\sqrt{\frac{3\left(1-v^{2}\right)}{2}} \cdot \frac{1}{t} \sqrt{\frac{\bar{P}}{t \cdot E}}
$$

The formulation proposed by Goland and Reissner does not take in account of the adhesive thickness. For joints with higher thickness, Hart-Smith proposed a correction for this $k$ coefficient.

According to Hart-Smith:

$$
k=\frac{1}{1+\lambda\left(\frac{l}{2}\right)+\frac{1}{6}\left(\lambda\left(\frac{l}{2}\right)\right)^{2}}
$$

With

$$
\lambda=\sqrt{12\left(1-v^{2}\right)} \frac{1}{t} \sqrt{\frac{\bar{P}}{t E}}
$$

The normal tension in the adherend would now be given by:

$$
P_{\text {max }}=\frac{\sigma_{y} \cdot b t}{1+3 k\left(1+\frac{t_{a}}{t}\right) b}
$$

Adams, Comyn, and Wake (1997) suggest that:

$$
\begin{aligned}
& \text { If } \frac{l}{t}<20 \text { than } k \approx 1 \Rightarrow P_{\text {max }} \approx \frac{\sigma_{y} \cdot b t}{4} \\
& \text { If } \frac{l}{t}>20 \text { than } k \approx 0 \Rightarrow P_{\text {max }} \approx \frac{\sigma_{y} \cdot b t}{1}
\end{aligned}
$$

From the previous expressions comes:

$$
\frac{l}{t}=\frac{25}{2}=12.5<20 \Rightarrow k \approx 1
$$

The maximum adherend failure load can be calculated simply by running an iteration process. This work intends to perform a practical validation of the criteria mentioned above, providing the reader with the capability to choose the criterion most suited for each application. The main goals of this experiment consist on the understanding the practical difficulties associated with the preparation and execution of a single overlap joint, and its standard procedures, on studying the influence of the surface preparation of the adherend in the joint resistance. In addition, this work also performs a comparison of the results of the different criteria used to predict the joint mechanical resistance with the experimental results.

\section{Materials and methods}

This section fully describes the specimens experimental tested, including information regarding specimen dimensions, material properties and manufacture procedures. 
The specimens tested in this work were single lap joints. Figure 5 shows the single lap joint configuration employed with all relevant dimensions marked, while Table 1 lists these dimensions and their values for the tested joints.

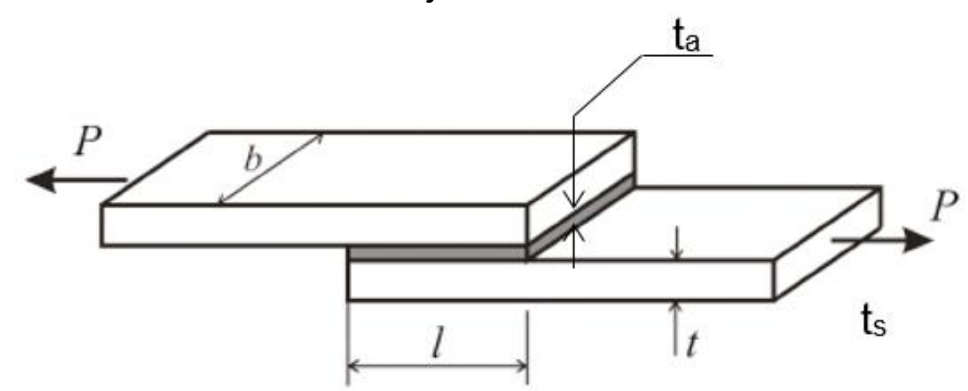

Figure 5: Representative illustration (da Silva, Öchsner, and Adams 2011) of an overlap joint and the specific nomenclature that will be used throughout the report

\begin{tabular}{cc}
\hline Specimen dimensions & Value \\
\hline Adherend thickness $\left(\boldsymbol{t}_{\boldsymbol{s}}\right)[\mathrm{mm}]$ & 2 \\
Adhesive thickness $\left(\boldsymbol{t}_{\boldsymbol{a}}\right)[\mathrm{mm}]$ & 0.2 \\
Width $(\boldsymbol{b})[\mathrm{mm}]$ & 25 \\
Overlap length $(\boldsymbol{l})[\mathrm{mm}]$ & 25 \\
\hline
\end{tabular}

Table 1: Main geometrical parameters of the SLJ specimen

The substrates are bonded with an acrylic adhesive, SikaFast ${ }^{\circ}$ - 5211 NT (SIKA AG, Baar, Switzerland). This is a fast curing, dual component adhesive, available in a double cartridge with two separate containers. Inside of the largest container is a thermosetting acrylic polymer that cures at room temperature when reacting with a catalyst hardener. The catalyst hardener is present in the second smaller container and it is mixed with a 10:1 volume ratio. Table 2 lists the main mechanical properties of this adhesive as stated in the manufacturer datasheet.

\begin{tabular}{cc}
\hline Property & Value \\
\hline Tensile yield stress $\left(\boldsymbol{\sigma}_{\boldsymbol{y}_{\boldsymbol{a}}}\right)[\mathrm{MPa}]$ & 10 \\
Shear yield stress $\left(\boldsymbol{\tau}_{\boldsymbol{y}_{\boldsymbol{a}}}\right)[\mathrm{MPa}]$ & 10 \\
Young's Modulus $\left(\mathrm{E}_{\mathrm{a}}\right)[\mathrm{MPa}]$ & 250 \\
Poisson's ratio $\left(\boldsymbol{v}_{\boldsymbol{a}}\right)$ & 0.35 \\
Ultimate elongation $\left(\boldsymbol{\varepsilon}_{\boldsymbol{a}}\right)[\%]$ & 200 \\
\hline
\end{tabular}

Table 2: Mechanical properties of the adhesive used (SikaFast 5211 NT)

The substrates used in the construction of the SLJ are composed of a mild steel, with the designation of Din ST.33. Table 3 lists the main mechanical properties of this material.

\begin{tabular}{cc}
\hline Property & Value \\
\hline Tensile yield stress $\left(\boldsymbol{\sigma}_{\boldsymbol{y}_{\boldsymbol{m}}}\right)[\mathrm{MPa}]$ & 185 \\
Young's Modulus $\left(\mathbf{E}_{\mathbf{a}}\right)[\mathrm{GPa}]$ & 210 \\
Poisson's ratio $\left(\boldsymbol{\nu}_{\boldsymbol{a}}\right)$ & 0.25 \\
Ultimate elongation $\left(\boldsymbol{\varepsilon}_{\boldsymbol{a}}\right)[\%]$ & 28 \\
\hline
\end{tabular}

Table 3: Mechanical properties of the mild steel used (DIN St33)

Adhesive bonding is essentially a surface phenomenon and the preparation of the contact surface is a vital condition for avoiding adhesive failure of the joint. To assess this effect, two specimen configurations were prepared for use in this study, one configuration with a treated surface and a second configuration with no surface preparation.

For the treated specimens, the first manufacture step consisted in cleaning the zone to be bonded with acetone. This was performed to remove all grease, dust, oils and lubricants that 
might exist. After, the same surface was manually sanded with fine grain sandpaper. The aim of this step was to remove the contamination layer (adsorbed gases, weakly bonded oxides, etc.) that decreases the interface energy. Sanding was performed in two perpendicular directions, shown in Figure 6, to ensure that the surface is uniformly sanded. After sanding, the surface was cleaned with acetone and scrubbed with absorbent paper to remove the ceramic dust and degrease the surface.

The untreated specimens were not subjected to any type of preparation and therefore were used in the "as supplied state".

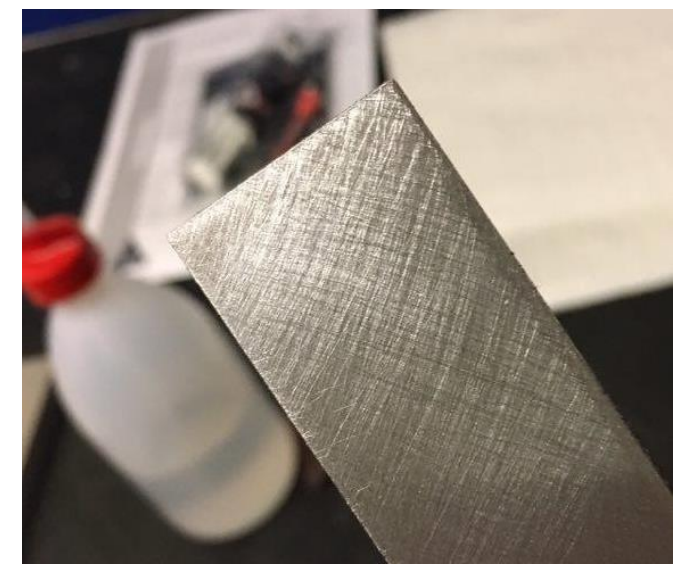

Figure 6: Pattern of $+45^{\circ}$ and $-45^{\circ}$ on the adherend

After preparing the surface, the adherends were correctly aligned with in the metallic mould, shown in Figure 7. Since the mould, shims and pins are metallic, it is important to apply a release agent. Otherwise, in the case of adhesive overflow the specimens would be bonded to the mould and the ensuing removal process could easily damage the specimens and the mould surface.

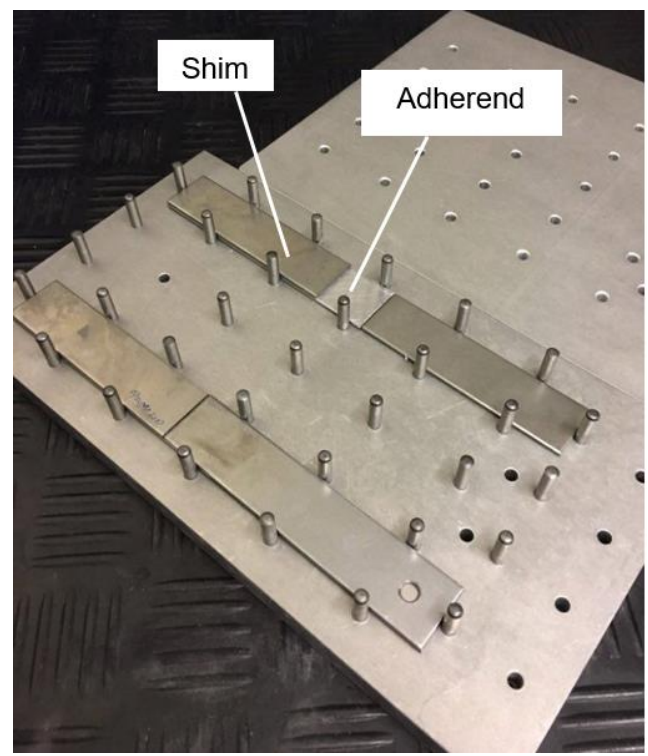

Figure 7: Image of the mould used with two specimens in place. The specimen on the left is not closed while the one on the right is closed and complete

Shims are devices that enable perfect control of the adhesive thickness, fillet geometry and overlap length (da Silva et al. 2008; da Silva et al. 2009). In this specific case, no fillets were created. The shim configuration used in this work is shown in Figure 8. 


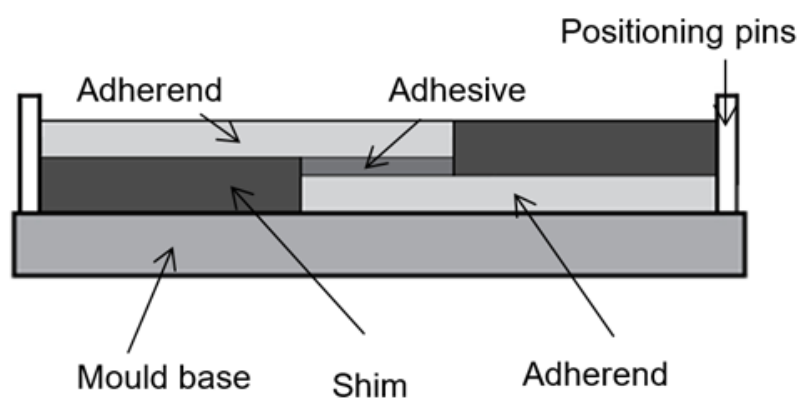

Figure 8: Schematic representation of the shim position and function

To mix the two-part adhesive, there is no need to use pneumatic applicator guns or centrifugal mixers to ensure a void free adhesive. The mixture of the two components is done automatically in the mixing tip. To ensure a perfect mixture, the first few grams of mixed adhesive were not used for bonding.

The adhesive was applied with the shim, lower adherend and positioning blocks already in the mould. The upper adherend was placed over the adhesive. The adhesive application process must be quick, as the open life of the adhesive is only about 3 minutes. The adhesive was applied in an alternating pattern to minimize air entrapment (Figure 9). The mould was then closed and a $10 \mathrm{Kg}$ weight was used to apply pressure on the specimens.

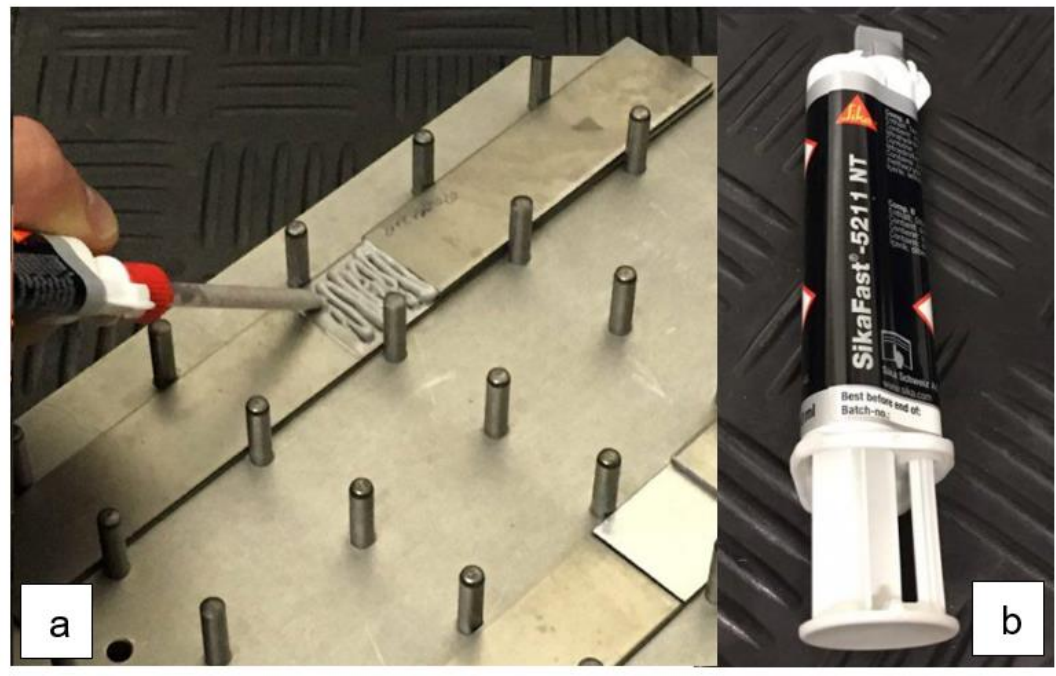

Figure 9: a) pattern of adhesive application b) two-part adhesive cartridge (containing resin and hardener) used

This pressure application procedure ensures that there is no free movement of the substrates during the cure process.

The cure parameters used were:

- Temperature - Room temperature (between $15^{\circ} \mathrm{C}$ and $25^{\circ} \mathrm{C}$ )

- Time - $14 \mathrm{~min}$ (recommended by the manufacturer for room temperature curing)

After the curing process, the specimens were carefully removed from the mould and separated from the spacers. The adhesive excess was manually removed with a file, reducing variability and enabling direct comparison of their results. The specimens were then tested in an INSTRON ${ }^{\circledR}$ (Norwood, MA, USA) universal testing machine (shown in Figure 10). The tests were made at a crosshead speed of $2 \mathrm{~mm} / \mathrm{min}$ speed, which approximates a quasi-static loading condition. 


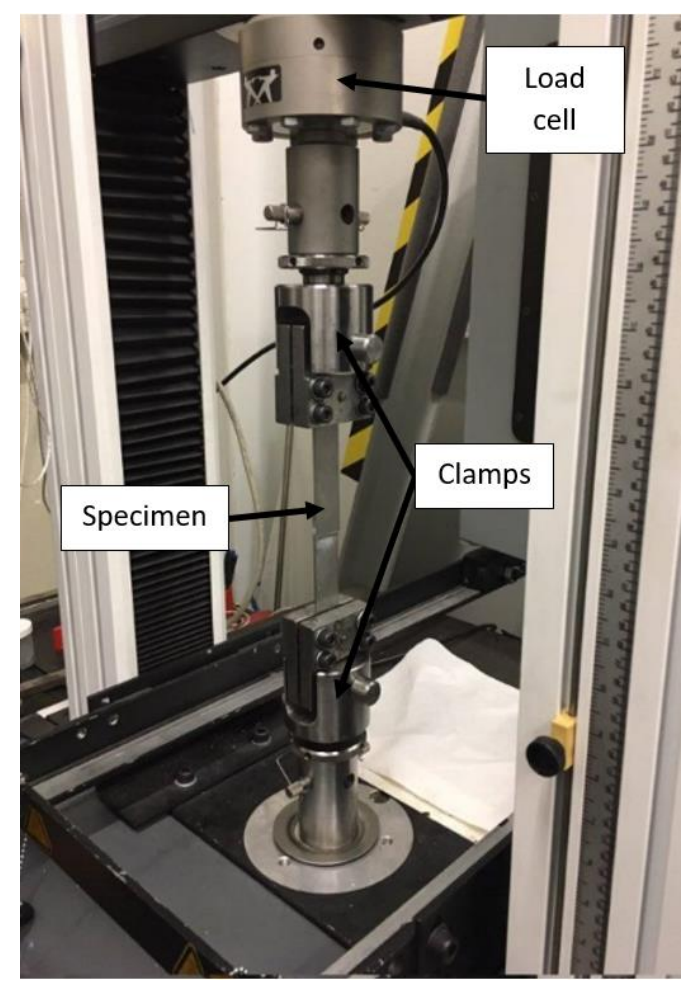

Figure 10: Universal testing machine used to perform the tension tests with main components indicated

\section{Results}

\subsection{Experimental results}

After completion of mechanical testing, the load-displacement curves were plotted for each of the specimens. Figure 11 shows representative load displacement curves for specimens with and without treated surface.

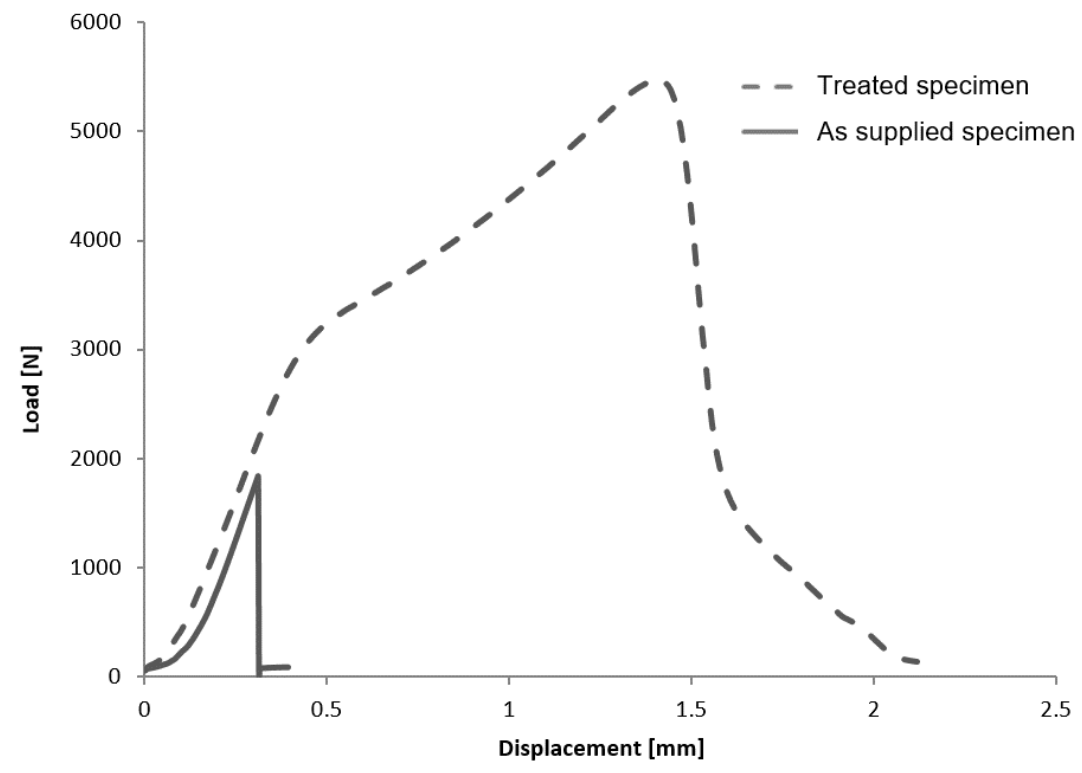

Figure 11: Representative load displacement curves for the two types of specimen tested (with treated surface and in supplied state)

From this data analysis, it becomes evident that the surface preparation has a great influence on joint strength. The absence of surface treatment decreased the joint resistance more than 4 times (calculated by comparing the two peaks in Figure 11). There is a clear nonlinear 
deformation zone mainly due to the plastic deformation of the adhesive. Even though the adherend plastically didn't yield, its elastic deformation produced a more disadvantageous stress state (bending moment), which significantly contributed to the premature failure of the adhesive. The representative fracture surfaces of the specimens are shown in Figure 12.
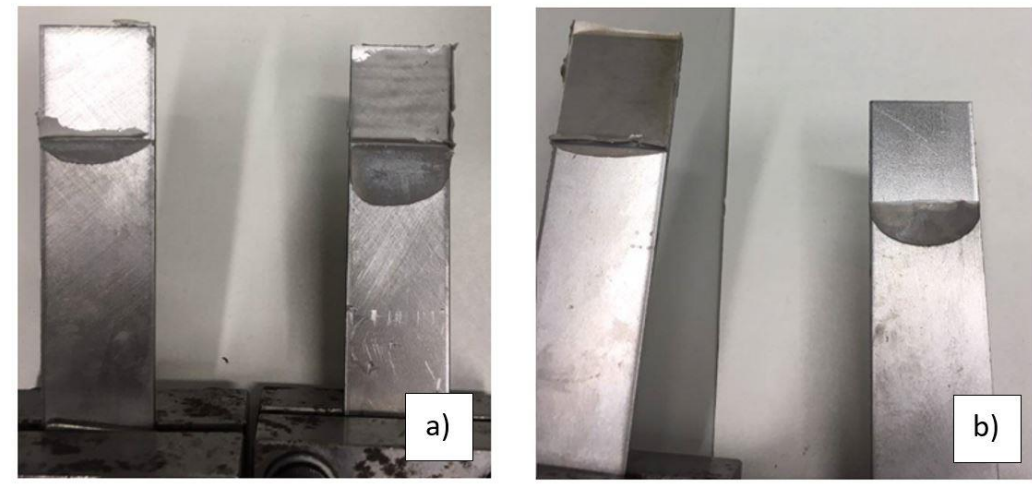

Figure 12: Aspect of the specimens after traction tested. On the left is the surface treated specimen, a), and on the right the joint that was manufactured with the adherend as supplied b)

To the naked eye, all failures could be classified as adhesive failures. Which would indicate that the treatment applied to the two specimen configurations was not effective.

For the untreated specimens, that is true and completely expected. Since there was no treatment applied to its surface. However, this was not expected for the treated specimens, which were subjected to surface treatment. Nevertheless, the adhesive suffered noticeable plastic deformation, which can be indicated by the off-white colour compared with the original grey showed by the untreated specimens (visible on Figure 12). It is possible that the fracture was cohesive and a very thin film of adhesive remained on the other adherend. This assumption can only be confirmed by analysis with a scanning electron microscope, which due to logistic constrains, was not possible.

\subsection{Analytical results}

\section{Volkersen model}

The Volkersen model was used to plot the relation between the maximum allowed load, as a function of the position of the overlap length, Microsoft Excel ${ }^{\circledR}$ (Microsoft, Redmond WA, USA) was used. The plot is shown in Figure 13.

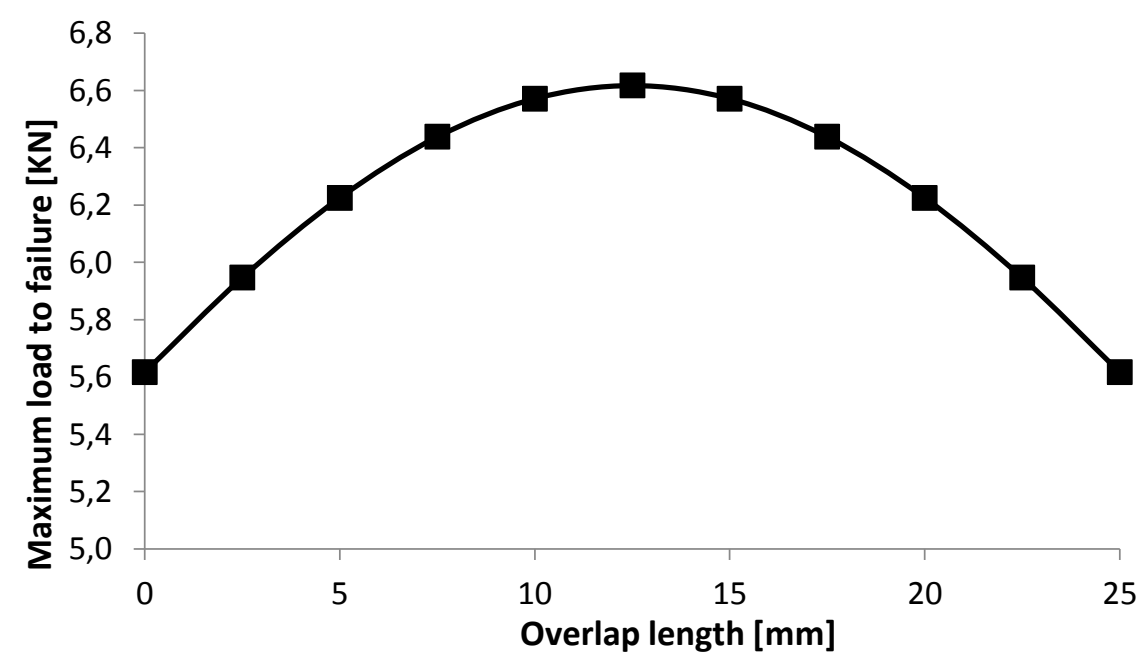

Figure 13: Distribution of the maximum load to failure throughout the overlap length, according to the Volkersen's shear lag model 
This plot allows the calculation of the failure load for the case under study:

$P \max \approx 5616.6[\mathrm{~N}]$, occurring at $x=0 \mathrm{~mm}$ and $\mathrm{x}=l=25 \mathrm{~mm}$

This model is obviously limited in utility as the Volkersen method does not take in account the possibility of plastic deformations. Even when comparing a highly alloyed steel with a carbon steel the plot difference would be small since the elastic modulus variation with the alloy elements is also small.

However, this model is suited for a quick analysis of the effect of overlap length on joint strength. It becomes clear that the longer the overlap length, the higher the joint strength. An increase of 2 times in the overlap length, yields an increase of 1.56 times in the joint strength.

$$
\frac{P_{\max }}{P x}=1.56
$$

As mentioned by Adams, Comyn, and Wake (1997), the increase of the overlap length is not the most efficient parametric optimization, being highly surpassed by the control of the overlap length. This control variable is also not studied in this paper but Figure 14 provides a qualitative visualization of the different possible responses.

Since the elongation $\varepsilon_{r}$ of the adhesive is very high ( $\left.\approx 200 \%\right)$, in other words it's far from being considered brittle, a large contrast between the experimental and theoretical results is expected.

Even in small amounts, the elongation in the plastic domain of the adhesive yields an increase in the failure load. So, the theoretical values should be considerably more conservative than the real values.

Lastly, it is important to note that the Volkersen's shear analysis takes no account of the noncollinear load directions. This yields a bending moment applied to the joint in addition to the in-plate tension. The rotation of the adherends will give rise to a geometrically non-linear problem. This specific phenomenon is better modelled by the Goland and Reissner Theory (Goland and Reissner 1944). For materials with low elastic modulus, like some composites, there have been some corrective modifications proposals to takes on account the adherends bending effects. Some authors (Zhu and Kedward 2005) usually employ what are known as TOM solutions (Tsai, Oplinger, and Morton 1998), which apply a correction to the shear lag model for single and double lap joints and assume that the shear stress is linear through the adherends.

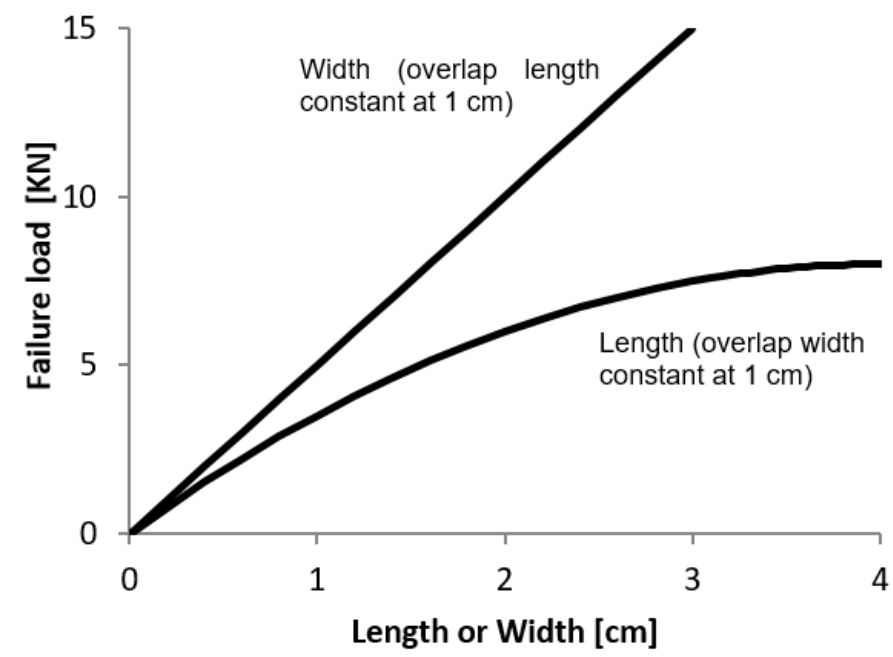

Figure 14: Effect of the overlap length and width on the failure load of a single overlap joint. Adapted from Pizzi and Mittal (2003) by the authors 


\section{Goland and Reissner model}

Using the Goland and Reissner model, a plot showing the distribution of the tension through the overlap length was created and is shown in Figure 15. Note that in this case the $x$ coordinate is considered to be zero at the midpoint of the overlap length.

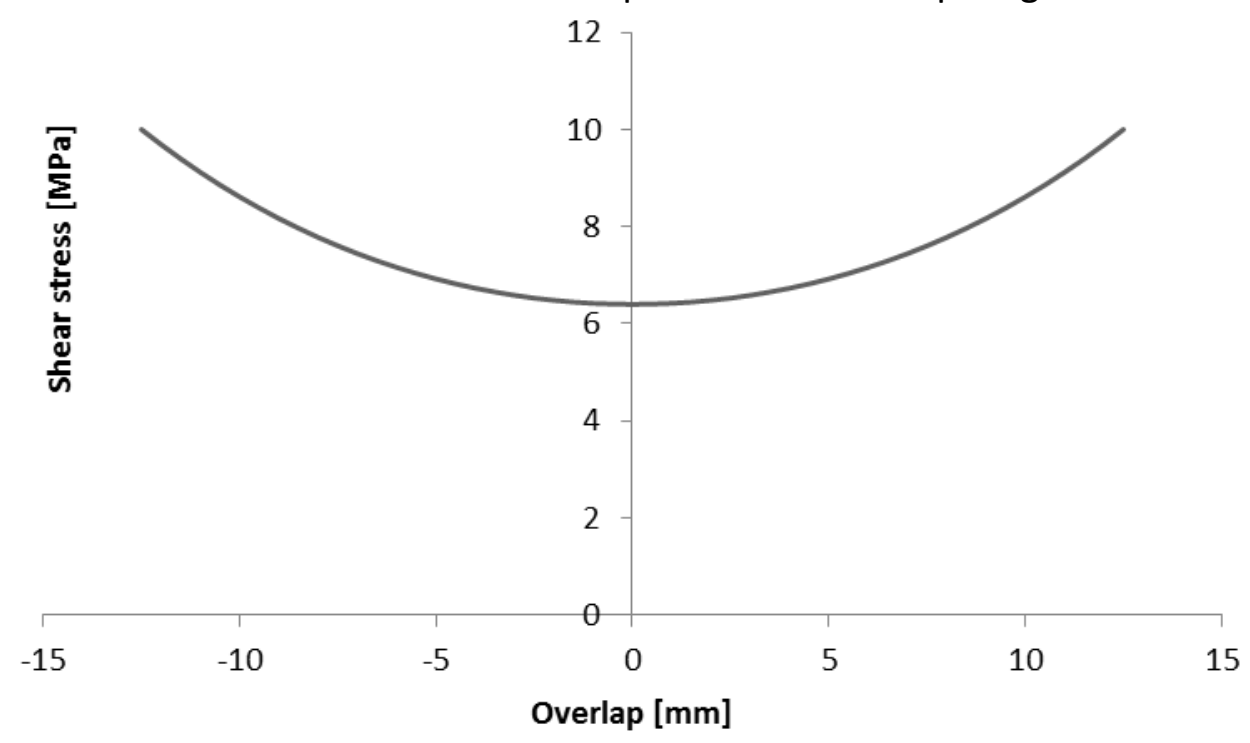

Figure 15: Distribution of the shear stress throughout the overlap length of the joint, calculated according to Goland and Reissner first theory

This calculation allows the estimation of the following maximum load:

\section{Generalized failure criterion}

$$
P_{\max } \approx 4716.5 \mathrm{~N}
$$

For ductile adhesives, the generalized failure criterion allows a quick estimation of the failure load, simply by multiplying the shear strength of the adhesive by the bonded area. According to this method, the failure load for the joints under study is:

$$
P_{\text {max }} \approx 6250.0 \mathrm{~N}
$$

\subsection{Adherend failure criterion}

Using the formulation proposed by Adams, Comyn, and Wake (1997), the maximum adherend failure load was calculated by running an iteration process. The failure loads predicted for each iteration are shown in Table 4 and Table 5.

\begin{tabular}{ccc}
\hline Iteration Number & $\mathbf{K}(\mathbf{2 2})$ & $\mathbf{P}_{\max }[\mathbf{N}](\mathbf{1 9 )}$ \\
\hline 1 & 1 & 2312.5 \\
2 & 0.763 & 8748.3 \\
3 & 0.627 & 8834.6 \\
4 & 0.625 & 8835.8 \\
5 & 0.625 & --- \\
\hline
\end{tabular}

Table 4: Goland and Reissner coefficients

\begin{tabular}{ccc}
\hline Iteration Number & $\mathbf{K}$ (22) & $\mathbf{P}_{\max }[\mathbf{N}] \mathbf{( 2 4 )}$ \\
\hline 1 & 1 & 2312.5 \\
2 & 0.753 & 8709 \\
3 & 0.601 & 8813.0 \\
4 & 0.599 & 8814.4 \\
5 & 0.599 & ---- \\
\hline
\end{tabular}

Table 5: Hart-Smith coefficients 


\subsection{Summary of predicted values}

In Table 6, a summary of all the theoretical estimated values is provided.

\begin{tabular}{cc}
\hline Criteria & Predicted failure load [N] \\
\hline Volkersen Analysis & 5616.6 \\
\hline Goland and Reissner theory & 4716.5 \\
Generalized Failure Criterion & 6250.0 \\
Adams, Comyn, and Wake & 8835.8 \\
(1997) (G\&R) & \\
Adams, Comyn, and Wake & 8814.4 \\
\hline
\end{tabular}

Table 6: Summary of analytically predicted failure loads

A final comparison between the theoretical and experimental results is shown in Figure 16. It is worth mentioning that the theoretical results can only be compared with the experimental results from the treated specimens, since the untreated specimens exhibited extremely low failure loads induced by adhesion failure.

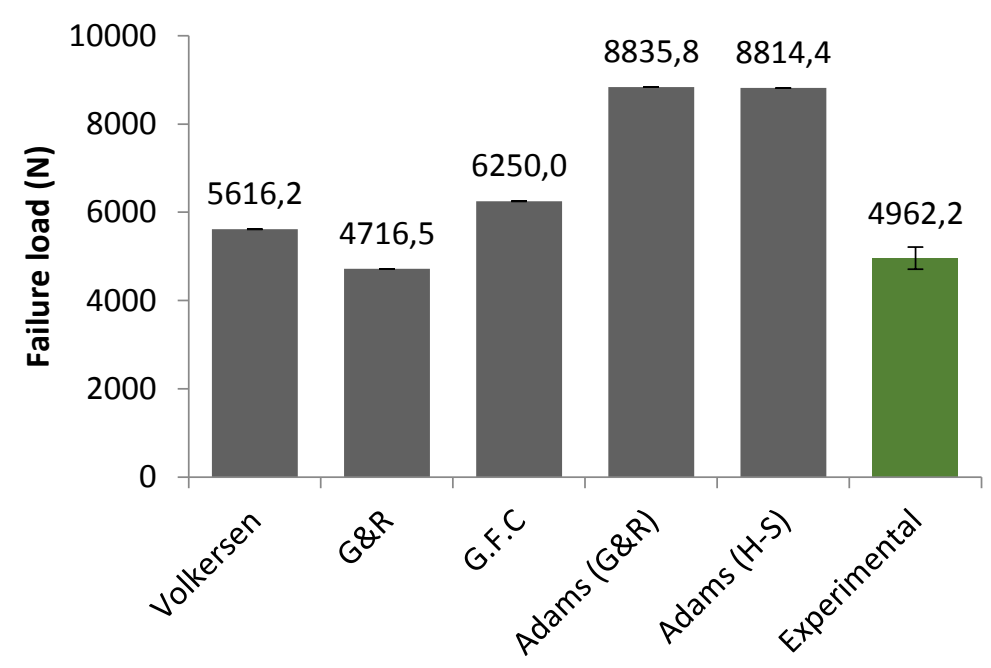

Figure 16: Comparison of all theoretical and experimental results. Experimental results correspond to the treated specimens

The two variants of the Adams, Comyn, and Wake (1997) theory applied were expected to overpredict a maximum failure and this indeed occurred. As the adhesive has a very ductile behaviour, the probability of the adherend being the weakest link was low. However, considering that the adherend was composed of mild steel, with a relatively low mechanical strength the adherend failure could still be a possibility.

Looking now for the Hart-Smith's model forecast, an over predicted estimation was also expected. Its inability for modelling the bending effect that happens during the test can be advanced as the main reason behind the model's inaccuracy. The assumption of entire plastic deformation on the overlap length can be validated by Figure 12a), where a uniform off-white colour can be seen.

The Generalized Failure Criterion was expected to suit this adhesive well given its ductility. However, the predicted result was slightly higher than the experimental value. This was probably due to the occurrence of adhesive failure.

Although the result obtained by the Volkersen theory seems reasonably accurate, this model cannot be understood as useful for the case under study. The fact that it does not take in account the bending effect over predicts the joint resistance. However, since it also models the adhesive as a brittle and elastic material, with null plastic deformation, the failure load 
would be under predicted. Its relative accuracy can therefore be attributed to a random combination of adhesive and adherend properties, and geometrical joint aspects.

Lastly, the Goland and Reissner model has provided a good, yet slightly conservative, prediction. This was expected as, in contrast to the Volkersen method, the Goland and Reissner theory does take into account to the bending moment, eliminating the previously described problem. In addition, since the Goland and Reissner theory evaluates not only the shear stress but also the peeling stress, a failure criterion was applied.

\section{Conclusions}

This work has demonstrated the extreme importance of surface treatment in the mechanical behaviour of an adhesive joint. Although both specimen types under test suffered adhesive failure, there was still a significant difference between treated and untreated specimens, highlighting the influence of the surface treatment. In this work, even specimens that were cleaned and mechanically treated still did not reach the full potential of the adhesive. A future work could focus on the improvement of the adhesion, by means of an alternative surface treatment method such as sandblasting or primer application.

Among all the analytical models used to predict joint strength, the Generalized Failure Criterion, the Goland and Reissner method and the Volkersen criterion were those closer to the experimental result. However, the Volkersen criterion is not applicable to the adhesive and adherends under study, so it cannot be considered as correct.

\section{References}

Adams, R. D., J. Comyn and W. C. Wake. 1997. Structural adhesive joints in engineering. Springer Netherlands.

da Silva, L. F., R. F. Lima, R. M. Teixeira and A. Puga. 2008. Closed-form solutions for adhesively bonded joints [Reports of the project "Development of the software for the design of adhesive joints"]. University of Porto, Portugal.

da Silva, Lucas F. M., R. J. C. Carbas, G. W. Critchlow, M. A. V. Figueiredo and K. Brown. 2009. "Effect of material, geometry, surface treatment and environment on the shear strength of single lap joints". International Journal of Adhesion and Adhesives 29 (6):621-632. Accessed June 20, 2017. DOI: 10.1016/j.ijadhadh.2009.02.012.

da Silva, L. F. M., A. G. de Magalhães and M. F. S. de Moura. 2007. Juntas adesivas estruturais. Porto: Publindústria, Edições Técnicas.

da Silva, Lucas F. M., Andreas Öchsner and Robert D. Adams. 2011. Handbook of Adhesion Technology. Springer Berlin Heidelberg. Accessed June 20, 2017. DOI: 10.1007/978-3-64201169-6.

Goland, M. and E. Reissner. 1944. "The stresses in cemented joints". Journal of applied mechanics 11 (1):A17-A27.

Gomes, Joaquim Francisco da Silva. 2004. Mecânica dos sólidos e resistência dos materiais. Porto: INEGI.

Hart-Smith, L. J. 1973. Adhesive-bonded double-lap joints: Technical report. Langley Research Center, National Aeronautics and Space Administration (NASA).

Pizzi, A. and K. L. Mittal. 2003. Handbook of adhesive technology, revised and expanded. CRC Press.

Tsai, M. Y., D. W. Oplinger and J. Morton. 1998. "Improved theoretical solutions for adhesive lap joints". International Journal of Solids and Structures 35 (12):1163-1185. Accessed June 20, 2017. DOI: 10.1016/S0020-7683(97)00097-8. 
Volkersen, Olaf. 1938. "Die Nietkraftverteilung in zugbeanspruchten Nietverbindungen mit konstanten Laschenquerschnitten". Luftfahrtforschung 15 (1/2):41-47.

Zhu, Y. and K. Kedward. 2005. Methods of analysis and failure predictions for adhesively bonded joints of uniform and variable bondline thickness. Office of Aviation Research, Federal Aviation Administration. 